\title{
Visual Halo
}

National Cancer Institute

\section{Source}

National Cancer Institute. Visual Halo. NCI Thesaurus. Code C50806.

Hazy ring around bright lights seen by some patients with refractive error or optical defects, e.g. cataracts, or corneal swelling. 